\title{
Atg12-Interacting Motif Is Crucial for E2-E3 Interaction in Plant Atg8 System
}

\author{
Kazuaki Matoba and Nobuo N. Noda* \\ Institute of Microbial Chemistry (BIKAKEN); 3-14-23 Kamiosaki, Shinagawa-ku, Tokyo 141-0021, Japan. \\ Received May 20, 2021; accepted June 26, 2021; advance publication released online July 1, 2021
}

\begin{abstract}
Autophagy is an intracellular degradation system regulating cellular homeostasis. The two ubiquitinlike modification systems named the Atg8 system and the Atg12 system are essential for autophagy. Atg8 and Atg12 are ubiquitin-like proteins covalently conjugated with a phosphatidylethanolamine (PE) and Atg5, respectively, via enzymatic reactions. The Atg8-PE conjugate binds to autophagic membranes and recruits various proteins through direct interaction, whereas the Atg12-Atg5 conjugate recognizes Atg3, the E2 enzyme for Atg8, and facilitates Atg8-PE conjugation by functioning as the E3 enzyme. Although structural and biochemical analyses have well established the Atg8-family interacting motif (AIM), studies on the interacting sequence for Atg12 are rare (only one example for human ATG12-ATG3), thereby making it challenging to define a binding motif. Here we determined the crystal structure of the plant ATG12b as a complex with the ATG12b-binding region of ATG3 and revealed that ATG12b recognizes the aspartic acid (Asp)-methionine (Met) motif in ATG3 via a hydrophobic pocket and a basic residue, which we confirmed critical for the complex formation by mutational analysis. This recognition mode is similar to that reported between human ATG12 and ATG3, suggesting that the Asp-Met sequence is a conserved Atg12-interacting motif (AIM12). These data suggest that AIM12 mediates E2-E3 interaction during Atg8 lipidation and provide structural basis for developing chemicals that regulate autophagy by targeting Atg12-family proteins.
\end{abstract}

Key words autophagy; Atg12-interacting motif; crystal structure; Atg8 system

\section{INTRODUCTION}

Autophagy is an intracellular degradation system conserved among eukaryotes. Autophagy induction generates a de novo double-membrane structure called an autophagosome, which sequesters cytoplasmic materials and delivers them to a lytic compartment (the lysosome in mammals and the vacuole in plants and yeasts) for degradation. ${ }^{1,2)}$ Amino acids obtained by protein degradation are transported from the lysosome/vacuole to the cytosol and recycled for protein synthesis. ${ }^{3)}$ Recent studies revealed that autophagy selectively degrades various materials, for example, organelles such as mitochondria and the endoplasmic reticulum, along with protein aggregates, phase-separated droplets, and even invasive microbes, thereby contributing to cellular homeostasis. ${ }^{4-6)}$ Pioneering studies using budding yeast identified two ubiquitin-like modification systems named the Atg8 system and the Atg12 system, which are evolutionarily conserved in higher eukaryotes (including mammals and plants) and essential for autophagy.,7) Atg8 and Atg12 are ubiquitin-like proteins covalently conjugated with phosphatidylethanolamine (PE) and Atg5, respectively, via enzymatic reactions. Atg7 functions as the E1-enzyme for both Atg8 and Atg12. After activating their C-terminal glycine, Atg7 transfers them to the catalytic cysteine of each E2 enzyme, Atg3 for Atg8 and Atg10 for Atg12. Atg12 (bound to Atg10 via a thioester) is then transferred to a lysine side-chain of Atg5, forming the Atg12-Atg5 conjugate without the help of E3 enzymes. ${ }^{8}$ The Atg12-Atg5 conjugate forms a complex with Atg16 and functions as the E3 enzyme for Atg8-PE conjugation: it activates Atg3 by rearranging its catalytic site while recruiting the $A \operatorname{tg} 3-A \operatorname{tg} 8$ thioester intermediate to membranes, thereby accelerating the transfer of Atg8 from
Atg3 to PE in the membranes. ${ }^{9-11)}$

Atg8-PE is tightly attached to autophagic membranes and plays critical roles in autophagy through interactions with various proteins. ${ }^{4)}$ The many structural analyses performed on Atg8 and its homologs as a complex with their binding partners have contributed to discovering the Atg8-family interacting motif (AIM), also known as the LC3-interacting region (LIR). Various selective autophagy receptors/adaptors recognize this motif. ${ }^{12,13)}$ By contrast, few Atg12-binding proteins are known, and only a human ATG12-ATG3 interaction structural analysis is available..$^{14)}$ Thus, the sequence that Atg12 recognizes has remained obscure.

Arabidopsis thaliana (At) conserves two Atg12 orthologs (AtATG12a and AtATG12b) and one Atg3 ortholog (AtATG3). ${ }^{15,16)}$ Here, we located the minimum binding region in AtATG3 for AtATG12b and determined the crystal structure of AtATG12b as a complex with the minimum binding region of AtATG3. Using structural information, we performed mutational analyses and identified essential residues for the AtATG12b-AtATG3 interaction. Structural comparison with the human ATG12-ATG3 complex allowed us to identify the consensus Atg12-interacting motif (AIM12), which mediates E2-E3 interaction during Atg8 lipidation.

\section{MATERIALS AND METHODS}

Preparation of Recombinant Proteins We performed plasmid construction and expression and protein purification (glutathione $S$ transferase (GST), GST-AtATG3s, Atg7, AtATG12b, GST-AtATG7 ${ }^{\mathrm{NTD}}$, and AtATG8a) as described previously. ${ }^{17)}$ We introduced the mutations leading to the indicated amino acid substitutions by PCR-mediated site-directed 
mutagenesis and confirmed the sequences by sequencing. We purchased AtATG3 ${ }^{\text {FR11 }}$ (residues 152-162) from Eurofins operon.

Crystallographic Analysis We obtained crystals of the AtATG12b-AtATG $3^{\text {FR11 }}$ complex using the sitting drop vapor diffusion method. We mixed and equilibrated a $4.6-7 \mathrm{mg} / \mathrm{mL}$ AtATG12b solution containing AtATG3 ${ }^{\text {FR11 }}$ (1:3 M ratio) with the reservoir solution $[6-8 \%(\mathrm{w} / \mathrm{v})$ PEG3350, $100 \mathrm{mM}$ citrate buffer $\mathrm{pH} 3.5-4.1]$ at $20^{\circ} \mathrm{C}$ (AtATG12b solution/reservoir solution volume ratio: $1: 1$ ). We supplemented the crystals with $20 \%$ glycerol (v/v), then flash-cooled them and kept them under a stream of nitrogen gas at $95 \mathrm{~K}$ during data collection. We collected diffraction data using a SPring-8 BL41XU and processed them with the HKL2000 program suite. ${ }^{18)} \mathrm{We}$ performed molecular replacement using MOLREP ${ }^{19)}$ in the $\mathrm{CCP}^{20)}$ software suite using the free AtATG12b structure (PDBID 1WZ3) as the search model. ${ }^{16}$ ) We built and refined the model using $\mathrm{COOT}^{21)}$ and phenix.refine ${ }^{22)}$ in the software suite PHENIX. ${ }^{23)}$ Inclusion of the twin operator enabled successful refinement. The Ramachandran plot, calculated using Rampage, ${ }^{24)}$ showed that 98.2 and $1.8 \%$ residues were in the favored or allowed region, respectively, whereas no residue was in the outlier region. Table 1 presents the diffraction data and refinement statistics. We used PyMOL to produce all structural figures. ${ }^{25)}$ We deposited the crystal structure of the AtATG12b-AtATG3 ${ }^{\text {FR11 }}$ complex in the protein database (PDB) (accession code 7EU4).

In Vitro Pull-Down Assay We performed an in vitro pull-down assay using COSMOGEL ${ }^{\circledR}$ GST-Accept gel (Nacalai Tesque, Inc., Kyoto, Japan) and Micro Bio-Spin ${ }^{\mathrm{TM}}$ Chromatography Columns (BIO-RAD, Hercules, CA, U.S.A.). We equilibrated $100 \mu \mathrm{L}$ of GST-Accept (50\% slurry) with phosphate-buffered saline (PBS). We incubated the proteins with GST-Accept for $1 \mathrm{~h}$ at $4{ }^{\circ} \mathrm{C}$. After washing the gel three times with $500 \mu \mathrm{L}$ of $\mathrm{PBS}$, we eluted the proteins with $40 \mu \mathrm{L}$

Table 1. Data Collection and Refinement Statistics

\begin{tabular}{|c|c|}
\hline & AtATG12b-AtATG3 $3^{\text {FR11 }}$ \\
\hline \multicolumn{2}{|l|}{ Data collection } \\
\hline Space group & $\mathrm{Pb}_{4}$ \\
\hline \multicolumn{2}{|l|}{ Cell dimensions } \\
\hline$a, b, c(\AA)$ & $128.47,128.47,163.17$ \\
\hline$\alpha, \beta, \gamma\left(^{\circ}\right)$ & $90.00,90.00,120.00$ \\
\hline Resolution $(\AA)$ & $45.96-3.20(3.31-3.20)$ \\
\hline$R_{\mathrm{sym}}$ & $0.144(0.767)$ \\
\hline$I / \sigma I$ & $7.1(2.1)$ \\
\hline Completeness $(\%)$ & $99.36(94.99)$ \\
\hline Redundancy & $3.4(3.1)$ \\
\hline \multicolumn{2}{|l|}{ Refinement } \\
\hline Resolution $(\AA)$ & $45.96-3.20$ \\
\hline No. reflections & 23805 \\
\hline$R_{\text {work }} / R_{\text {free }}$ & $0.238 / 0.285$ \\
\hline \multicolumn{2}{|l|}{ No. atoms } \\
\hline Protein & 9305 \\
\hline \multicolumn{2}{|l|}{$B$-factors } \\
\hline Protein $\left(\AA^{2}\right)$ & 77.3 \\
\hline \multicolumn{2}{|l|}{ R.m.s. deviations } \\
\hline Bond lengths $(\AA)$ & 0.009 \\
\hline Bond angles $\left({ }^{\circ}\right)$ & 1.41 \\
\hline
\end{tabular}

Values in parentheses are for highest-resolution shell. of Buffer E (50 mM Tris- $\mathrm{HCl}$ pH 7.5, $10 \mathrm{mM}$ reduced glutathione). We subjected the eluate to sodium dodecyl sulfate-polyacrylamide gel electrophoresis (SDS-PAGE) for Coomassie Brilliant Blue staining and Western blotting to identify the bound proteins. We performed chemiluminescent detection using Immobilon Western Chemiluminescent horseradish peroxidase (HRP) Substrate (Millipore) and ImageQuant LAS 4000 mini (GE Healthcare, Chicago, IL, U.S.A.). Dr. Ohsumi's laboratory provided the anti-AtATG12b antibody. ${ }^{16}$ ) We purchased the anti-DDDDK-tag monoclonal antibody (mAb)-HRP-DirecT (Monoclonal, FLA-1) from MBL and the anti-Rabbit immunoglobulin G (IgG) HRP Conjugate (W4011) from Promega (Madison, WI, U.S.A.). We used the same protocol for the competitive binding assay.

Isothermal Titration Calorimetry (ITC) We exchanged the solvent of all proteins with the same lot of PBS using a HiPrep 26/10 Desalting column (GE Healthcare). We performed ITC experiments using a Microcal iTC200 calorimeter (Malvern Panalytical), stirring at $1000 \mathrm{rpm}$. We maintained the temperature at $25^{\circ} \mathrm{C}$ and set the reference power to 5 . To titrate the samples, we pre-injected $0.4 \mu \mathrm{L}$ and performed 18 $2-\mu \mathrm{L}$ injections at 120 -s intervals into a cell. Protein concentrations at cell and syringe are as follows: wild-type AtATG3 $133 \mu \mathrm{M} v s$. AtATG12b $11.6 \mu \mathrm{M}$, AtATG3 ${ }^{\mathrm{FR}}$ (residues 88-188) $168 \mu \mathrm{M} v s$. AtATG12b $13.9 \mu \mathrm{M}$, AtATG3 D152A $118 \mu \mathrm{M} v s$. AtATG12b $13.9 \mu \mathrm{M}$, I154A $51 \mu \mathrm{M} v s$. AtATG12b $11.9 \mu \mathrm{M}$, D156A $102 \mu \mathrm{M} v s$. AtATG12b $7.8 \mu \mathrm{M}$, M157A $69 \mu \mathrm{M} v s$. AtATG12b $21.2 \mu \mathrm{M}$, E158A $77 \mu \mathrm{M} v s$. AtATG12b $11.6 \mu \mathrm{M}$. We analyzed titration data using MicroCal Origin 7.0 software to determine the enthalpy $(\Delta \mathrm{H})$, dissociation constant $\left(K_{\mathrm{d}}\right)$, and stoichiometry of binding $(\mathrm{N})$. The error of each parameter indicates the fitting error.

\section{RESULTS}

Identification of the Minimum AtATG12b-Binding Region in AtATG3 Structural studies on budding yeast Atg3 revealed that Atg3 possesses an intrinsically disordered region called the flexible region (FR) that is inserted into the E2 core fold. ${ }^{26)}$ The Atg3 FR, consisting of 80-100 residues, is conserved among Atg3 homologs, including human and plant ATG3, and interacts with Atg7 and the Atg12-Atg5 conjugate. $^{14,26-29)}$ The AtATG3 crystal structure $^{30}$ ) showed a disordered approx. 100-residue region (residues 87-189) corresponding to the FR (Fig. 1A). Moreover, AtATG3 directly binds to AtATG12b in vitro. ${ }^{31)}$ However, the AtATG3 region responsible for the interaction remained undetermined. We first performed an in vitro pull-down assay between GSTAtATG3 and AtATG12b and showed that AtATG3 directly binds to AtATG12b in a manner depending on the FR (Fig. $1 \mathrm{~B}$, lanes wild type (WT) and $\triangle F R$ ). Thus, similarly to yeast and human homologs, the AtATG3 FR interacts with AtATG12b.

To determine the binding affinity, we performed ITC experiments. The dissociation constants between AtATG12b and full-length AtATG3 and its FR (residues 88-188) alone were 2.4 and $3.8 \mu \mathrm{M}$, respectively, confirming that the FR is the major AtATG12b-binding region in AtATG3 (Fig. 1C). We compared the Homo sapiens (Hs) ATG3 and AtATG3 FR sequences and found an approx. 30-residue region located at the C-terminal portion with a relatively high sequence homology 
A.

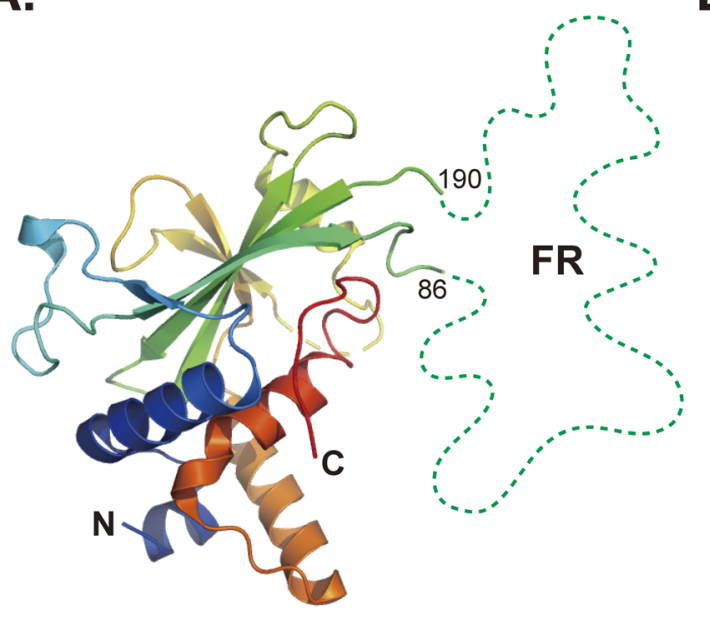

B.

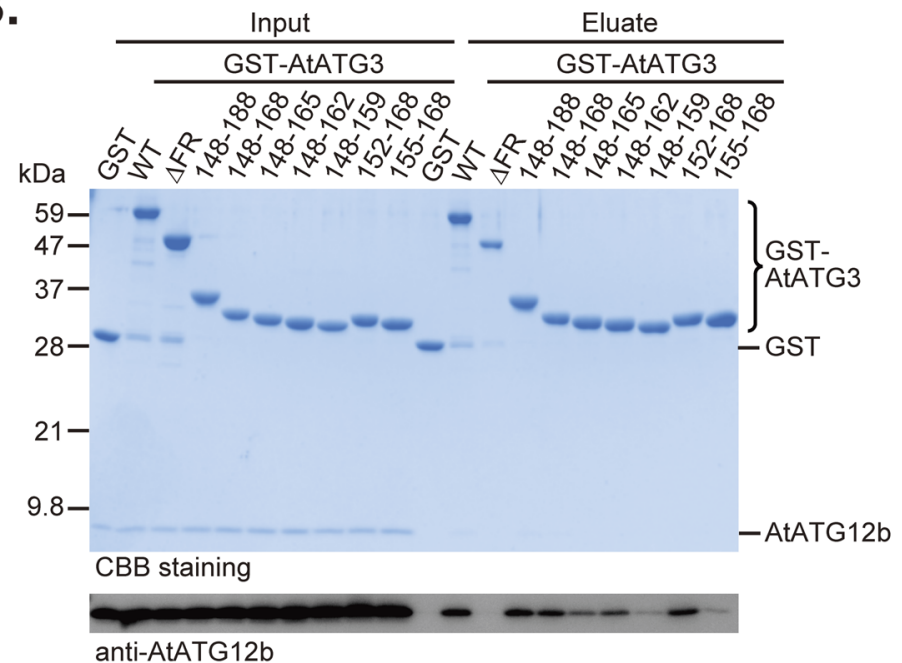

C. AtATG3 WT
vs AtATG12b AtATG3 FR

vs AtATG12b

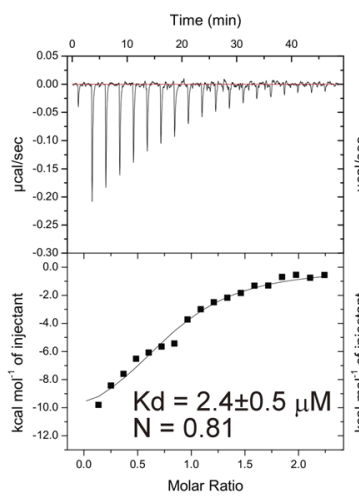

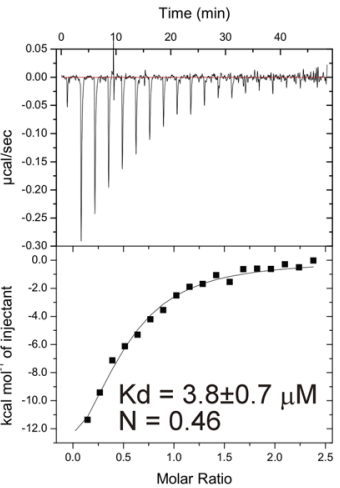

D.

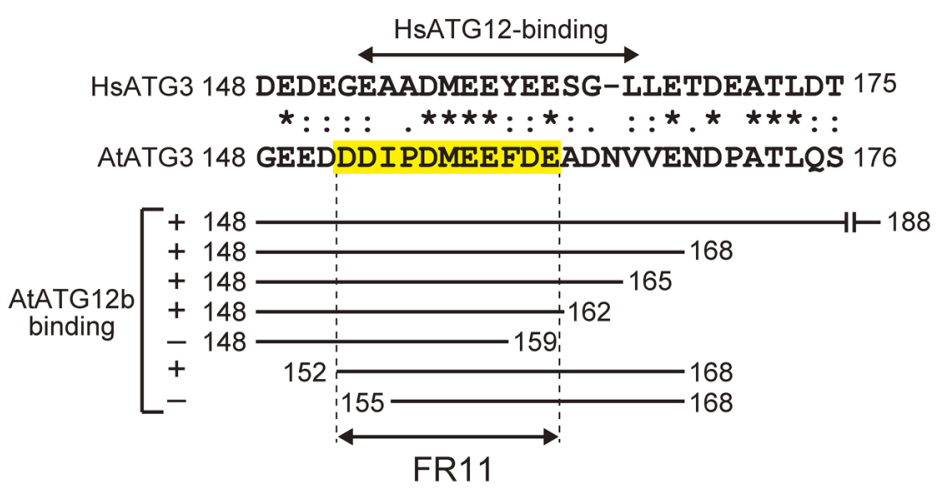

Fig. 1. Identification of the Minimum AtATG12b-Binding Region in AtATG3

Panel A AtATG3 crystal structure. Only the AtATG3 portion in the AtATG3-AtATG7 complex structure (PDB 3VX8) is shown. N and C indicate the N- and Cterminus. The broken line indicates the FR. Panel B In vitro pull-down assay between GST-AtATG3 and AtATG12b. Panel C Binding affinities between AtATG3 and AtATG12b determined by ITC. Panel D Sequence alignment of the highly conserved FR portion between HsATG3 and AtATG3 prepared using ClustalW. ${ }^{35)}$ Asterisks, colons, and periods indicate identical, highly similar, and similar amino acids, respectively. Below the alignment is the summary of the in vitro pull-down assay shown in Panel B. (Color figure can be accessed in the online version.)

(Fig. 1D, top). The in vitro pull-down assays on FR fragments suggested that the 11-residue sequence (residues 152-162; termed FR11) in AtATG3, which overlaps with the HsATG12 binding region in HsATG3 (Figs. 1B, D, bottom), is the minimum AtATG12b-binding region. ${ }^{14)}$

Crystal Structure of AtATG12b Complexed with AtATG3 $^{\text {FR11 }}$ To reveal the interaction mode between AtATG12b and AtATG3, we crystallized a mixture of AtATG12b and AtATG $3^{\text {FR11 }}$ and obtained a $3.2 \AA$ resolution crystal structure (Table 1). The asymmetric unit of the crystal contained 14 AtATG12b molecules, which formed seven intertwined dimers (residues 61-94 from one protomer fold into a ubiquitin fold together with the residues 1-60 from another protomer within the dimer), similar to the previously reported AtATG12b structure $^{16)}$ (Supplementary Figs. S1A, B). Besides, four AtATG12b molecules formed complexes with AtATG3 ${ }^{\text {FR } 11}$ molecules in a similar manner (Figs. S1C, D). Structural comparison with free AtATG12b suggests that AtATG3 ${ }^{\text {FR11 }}$ binding did not affect the conformation of AtATG12b (Fig. S1E).

AtATG12b has a ubiquitin fold consisting of four $\beta$-strands $(\beta 1-\beta 4)$ and two $\alpha$-helices $(\alpha 1, \alpha 2)$. AtATG3 ${ }^{\mathrm{FR} 11}$ binds to $\beta 2$ and $\alpha 1$ (Fig. 2A). The AtATG3 ${ }^{\text {FR11 }}$ residue most obviously involved in the interaction is Met157. Its side-chain is buried into the hydrophobic pocket formed by $\beta 2$ and $\alpha 1$ of AtATG12b. This hydrophobic pocket, named the M-site, consists of Val14, Leu16, Phe30, Val32, Val41, and Phe44 (Fig. 2B). The M-site is half-surrounded by basic residues, including Lys11 and Lys31, which are respectively close to Asp156 and Asp153 of AtATG3 ${ }^{\text {FR11 }}$ (Figs. 2A, right, 2B). Besides, the Ile154 side-chain forms hydrophobic interactions with that of Val13 and the aliphatic portions of Lys29 and Lys31, and the AtATG $3^{\text {FR11 }}$ backbone forms two hydrogen bonds with the Lys31 backbone.

Structure-Based Mutational Analysis Reveals Critical AtATG3 Residues for AtATG12b Binding To study the importance of the interactions observed in the AtATG12bAtATG $3^{\text {FR11 }}$ complex structure, we introduced alanine (Ala) substitutions at the AtATG12b binding region in GSTAtATG3 and studied their interaction with AtATG12 in an in vitro pull-down assay. As shown in Fig. 3A, substituting 
A.
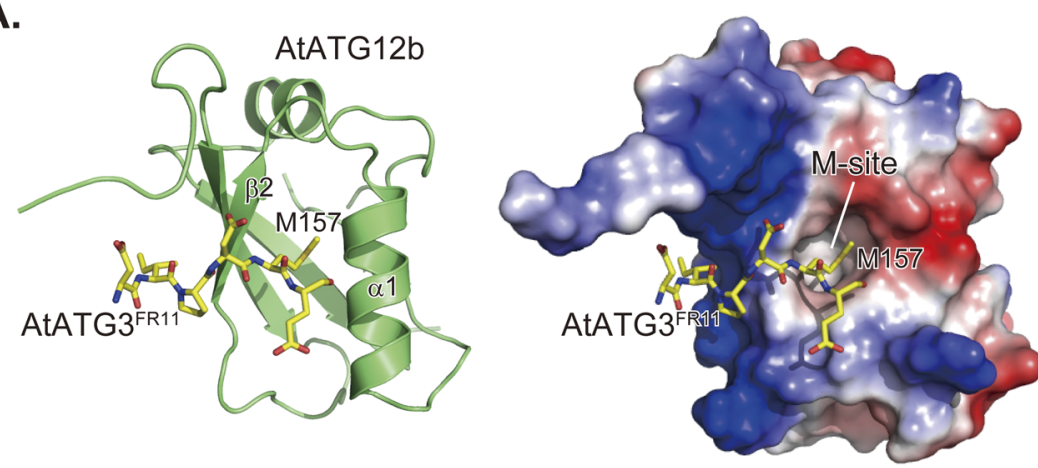

B.
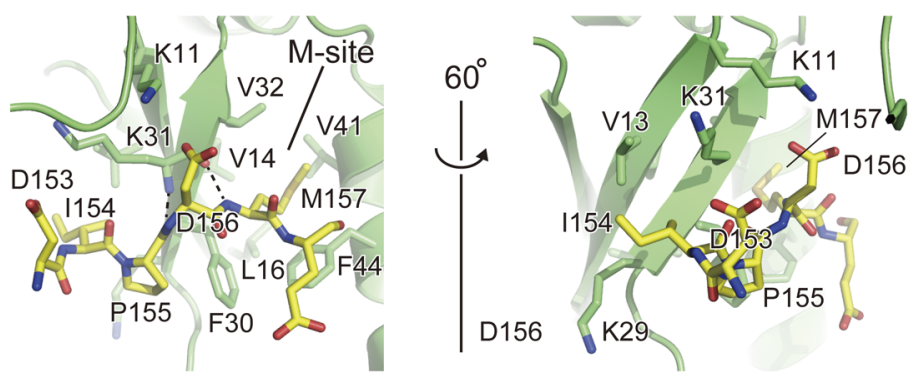

Fig. 2. Crystal Structure of the AtATG12b-AtATG3 ${ }^{\text {FR } 11}$ Complex

Panel A Overall structure of the AtATG12b-AtATG3 ${ }^{\text {FR11 }}$ complex. Left, AtATG12b is shown as a ribbon model (left) and a surface model colored based on the electrostatic potential (right), where blue and red indicate positive and negative charges. AtATG $3^{\mathrm{FR} 11}$ is shown as a stick model, with carbon, oxygen, nitrogen, and sulfur atoms in yellow, red, blue, and orange, respectively. Panel B Close-up view of the AtATG12b-AtATG3 ${ }^{\mathrm{FR} 11}$ interaction. Coloring is as in Panel A. Broken lines indicate hydrogen bonds between the AtATG12 and AtATG ${ }^{\mathrm{FR} 11}$ backbones. (Color figure can be accessed in the online version.)

A.

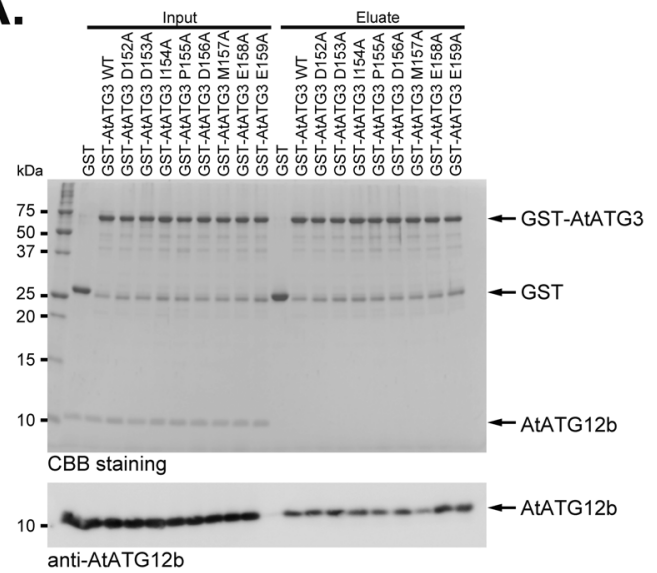

C.

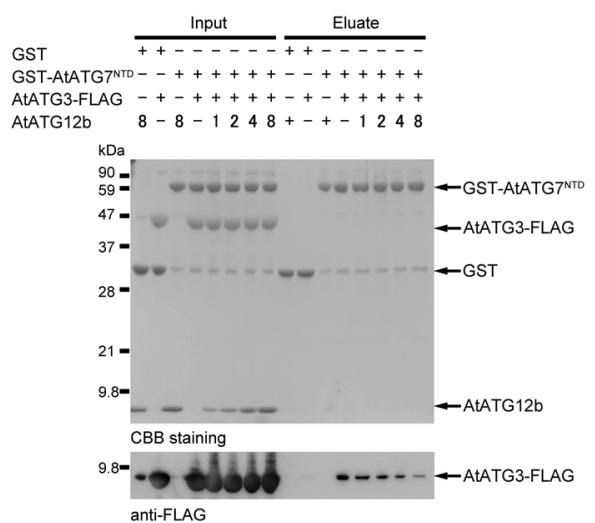

B.

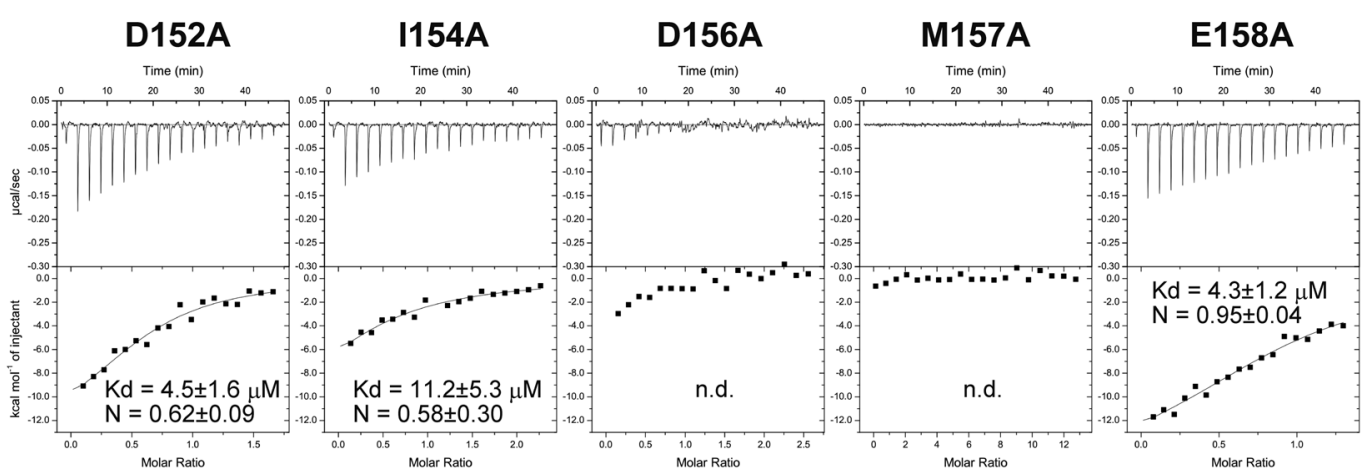

Fig. 3. Effect of Mutations on the AtATG12b-AtATG3 Interaction Using ITC

Panel A In vitro pull-down assay between GST-AtATG3 mutants and AtATG12b. Panel B Binding affinities between AtATG3 mutants and AtATG12b determined by ITC. Panel C In vitro pull-down assay between the NTD of AtATG7 fused with GST (GST-AtAtg $7^{\text {NTD}}$; at $5 \mu$ M) and AtATG3-FLAG (at $6.8 \mu$ M) with or without AtATG12b. Numbers 1, 2, 4, and 8 in the AtATG12b line indicate the AtATG12b concentration of 4.3, 8.6, 17.2, and 34.4, respectively. 
Met157 with an Ala (M157A mutation) most severely impaired the interaction with AtATG12b. To analyze the mutational effect more quantitatively and accurately, we then performed ITC experiments using AtATG12b and AtATG3 mutants (Fig. 3B). No thermal change occurred when titrating the AtATG3 M157A mutant, indicating that Met157 is the most critical residue for the interaction with AtATG12b. The D156A mutation severely impaired the interaction, the I154A mutation had a mild effect, whereas the D152A or D158A mutations only slightly reduced the interaction. These results are consistent with the interactions observed in the crystal and suggest that the Asp156-Met157 sequence is crucial for binding to AtATG12b.

Yeast Atg3 binds to the N-terminal domain (NTD) of Atg7 through its FR. ${ }^{26,27,32,33)}$ Moreover, human ATG7 (E1 enzyme) and ATG12-ATG5 (E3 enzyme) competitively bind to ATG3 (E2 enzyme). ${ }^{28)}$ These data suggest that the Atg3 FR is the common binding site for Atg7 and Atg12 and that the Atg3 FR-Atg7 and Atg3 FR-Atg12 interactions are exclusive to each other. We studied whether this competitive interaction also occurs with plant homologs using an in vitro pull-down assay. GST-fused AtATG7 ${ }^{\text {NTD }}$ directly bound to AtATG3, and AtATG12b dose-dependently impaired this interaction (Fig. 3C). These data suggest that AtATG12b and AtATG7 compete for binding to the AtATG3 FR and that this competitive interaction is conserved through evolution.

Structural Comparison of the Plant and Human ATG12-ATG3 Complexes Reveals the Core Sequence of the Atg12-Interacting Motif Besides the plant structure reported here, the ATG12-ATG3 complex has been reported for human homologs as a complex between the HsATG3 FR fragment (RIA12, residues 140-170) and the HsATG12-HsATG5 conjugate bound to a fragment of HsATG16L1. ${ }^{14)}$ Figure 4A shows a superimposition of these structures on the ATG12 moiety. Plant and human ATG12 assume a similar ubiquitin fold with a hydrophobic pocket between $\alpha 1$ and $\beta 2$, which we called the M-site above. Among the 31 HsATG $3^{\text {RIA12 }}$ residues, residues 153-165 showed defined electron density and were modeled as a helix and its N-terminal extended tail (Fig. 4A). In the modeled region, the N-terminal tail (residues 153-157) of HsATG3 ${ }^{\text {RIA12 }}$ had a conformation similar to that of AtATG ${ }^{\text {FR11 }}$ residues $153-157$ and was bound to the same part of ATG12. Notably, the side-chain of Met157 of both HsATG3 and AtATG3 bound to the M-site of ATG12 similarly. Furthermore, the side-chain of Asp156 of both HsATG3 and AtATG3 was close to that of Lys54 (HsATG12) or its equivalent Lys11 (AtATG12b). Hydrogen bonds between the backbones of ATG3 and ATG12 were also conserved. By contrast, the other interactions between ATG3 and ATG12 were not conserved between human and plant complex structures. In the human complex, ATG3 residues 158-174 folded into an $\alpha$-helix and the Glu158 and Glu161 side-chains formed electrostatic interactions with those of Lys54 and Arg79 of ATG12, respectively. Meanwhile, in the plant complex, ATG3 formed no $\alpha$-helix and no equivalent interactions occurred. One amino acid difference between human and plant ATG12 may explain this: HsATG12 Arg79 corresponds to AtATG12b Asp36, which repulses the acidic AtATG3 residues. Conversely, the interactions observed between AtATG3 Asp153 and AtATG12b Lys31, and between AtATG3 Ile154 and AtATG12b Val13 had no equivalent in the human complex, which can also be attrib-
A.

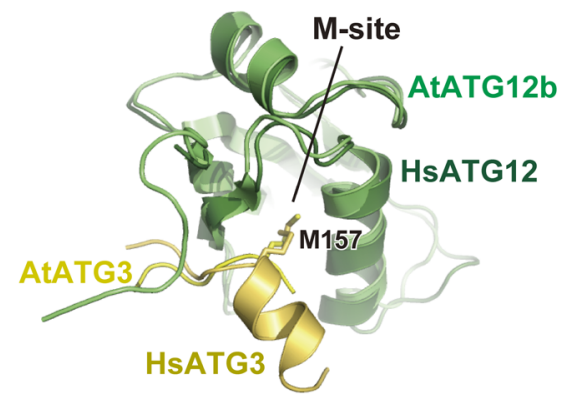

B.
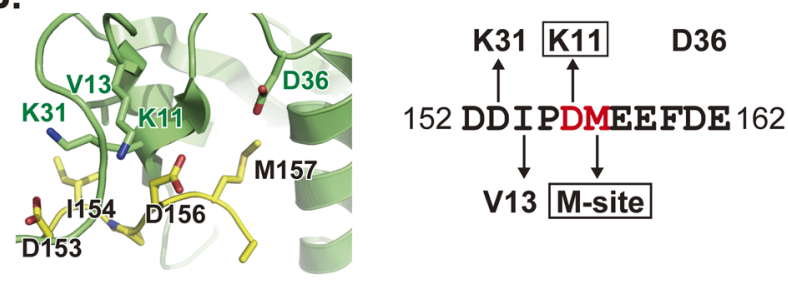

C.
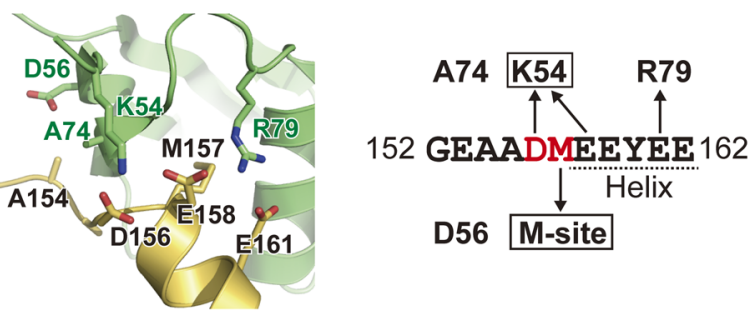

D.
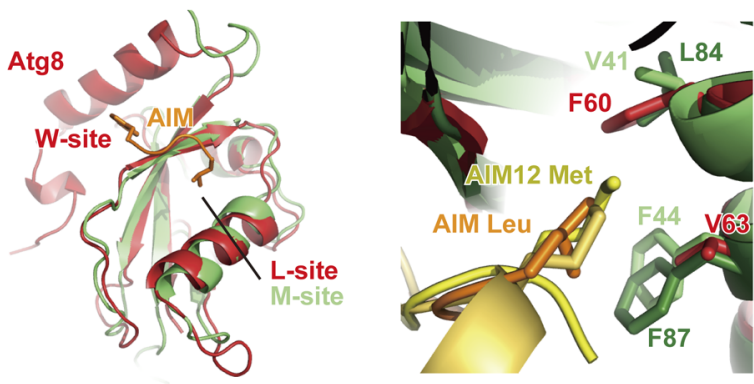

Fig. 4. Structural Comparison Suggests a Consensus Binding Motif

Panel A Superimposition of the HsATG12-HsATG3 ${ }^{\text {RIA12 }}$ portion of the HsATG12-HsATG5-HsATG16L1 fragment complex structure (PDB 4NAW) onto the HsATG12b-HsATG3 ${ }^{\mathrm{FR} 11}$ complex structure. Panel B Close-up view of the interaction between AtATG12b and AtATG3 ${ }^{\mathrm{FR} 11}$ (left) and summary of the main interactions (right). Panel C Close-up view of the interaction between HsATG12 and HsATG $^{\text {RIA12 }}$ (left) and summary of the main interactions (right). Panel D Superimposition of the Atg8-AIM complex structure (PDB 2ZPN) on the AtATG12bAtATG $3^{\text {FR11 }}$ complex structure (left). Trp and Leu in the AIM are bound to the $\mathrm{W}$-site and L-site in Atg8, respectively. Right shows a close-up view of the Msite/L-site. Dark and light green indicate HsATG12 and AtATG12b, respectively, whereas red indicates Atg8. (Color figure can be accessed in the online version.)

uted to amino acid differences in ATG12: AtATG12b Lys31 and Val13 correspond to HsATG12 Ala74 and Asp56, respectively. These data suggest that the common binding sequence in ATG3 for ATG12 is Asp156-Met157 and that some residues at both sides of the aspartic acid (Asp)-methionine (Met) motif, which are often acidic, form species-specific interactions with ATG12 to increase the binding affinity.

\section{DISCUSSION}

Protein-protein interactions mediate various cellular functions. They are often mediated by a conserved short sequence called a "motif," which endows diverse proteins with a spe- 
cific binding ability. Structural and biochemical analyses have identified various motifs in ubiquitin and ubiquitin-like proteins, including the AIM. Various autophagy-regulating proteins contain the AIM. This motif plays critical roles in autophagy by mediating the interaction with Atg8, an autophagy-specific ubiquitin-like protein. ${ }^{12,13)}$ Atg12 is another autophagy-specific ubiquitin-like protein. However, because of a lack of structural and biochemical studies on Atg12, the Atg12-interacting motif has remained undefined. In this study, we determined the crystal structure of plant ATG12 bound to a short ATG3 fragment and characterized their interaction using biochemical methods. By comparing with the previously reported human ATG12-ATG3 complex structure, we defined the short Asp-Met sequence as the core of the Atg12-interacting motif, which we abbreviated as AIM12. Among AIM12 residues, the most critical for the interaction with Atg12 is Met. It binds deeply into the M-site, a hydrophobic pocket at the center of the ubiquitin fold. Structurally, the M-site corresponds to the L-site in the ubiquitin fold of Atg8, which interacts with the AIM (Fig. 4D, left). The L-site favors leucine (Leu), isoleucine (Ile), and Val, whereas the M-site favors Met. The hydrophobic cavity shape differences between Atg12 and Atg8-family proteins explain this preference difference: in the M-site, residues Val41 and Phe44 in AtATG12b (Leu84 and Phe87 in HsATG12) correspond to Phe60 and Val63 in Atg8, respectively (Fig. 4D, right).

Lipidation of Atg8 proceeds via consecutive reactions catalyzed by E1-E2-E3 enzymes similarly with ubiquitination. Atg3 (E2) initially binds Atg7 (E1) and receives Atg8 from Atg7. Atg3 then changes the interacting partner from Atg7 to the Atg12-Atg5-Atg16 complex (E3), which enables Atg3 to transfer Atg8 to PE. Competitive interaction of FR with Atg7 and $\mathrm{Atg} 12^{28}$ (Fig. 3C) seems to enable these consecutive reactions. The simplicity of the core sequence of AIM12 would be advantageous for endowing FR with the Atg12-binding ability while keeping the Atg7-binding ability.

The Asp-Met sequence is too short to mediate the specificity for Atg12. Residues sandwiching the Asp-Met sequence, typically acidic residues, reinforce the interaction through non-conserved interactions with Atg12. Because these auxiliary interactions are not conserved between plant and human complex structures, defining a common binding motif besides the Asp-Met sequence is challenging. Multiple sequence alignment using Clustal Omega ${ }^{34)}$ shows that Atg3 homologs from various species, but not all, conserve the Asp-Met sequence in the FR (Supplementary Fig. S2). It would be interesting to know whether the Atg12-binding ability is conserved in the FR of Atg3 homologs lacking the Asp-Met sequence. Further studies are required to show whether AIM12 is utilized by proteins outside the Atg3-family and to further define the diversity and consensus of AIM12, which will provide us basis for developing chemicals that regulate autophagy through targeting Atg12-mediated interactions.

Acknowledgments We thank Drs. Yoshinori Ohsumi and Kohki Yoshimoto for providing AtATG genes and antibody against AtATG12b. The synchrotron radiation experiments were performed at BL41XU in SPring-8. This work was supported in part by JSPS KAKENHI Grant Number 25111004, 18H03989, 19H05707 (to N.N.N.), 25871076, 15K21608, 18K06097 (to K.M.), CREST, Japan Science and Technol- ogy Agency Grant number JPMJCR13M7, JPMJCR20E3 (to N.N.N.), and grants from the Takeda Science Foundation (to N.N.N.), and from Mochida Memorial Foundation for Medical and Pharmaceutical Research (to N.N.N.).

Conflict of Interest The authors declare no conflict of interest.

Supplementary Materials The online version of this article contains supplementary materials.

\section{REFERENCES}

1) Morishita H, Mizushima N. Diverse cellular roles of autophagy. Annu. Rev. Cell Dev. Biol., 35, 453-475 (2019).

2) Nakatogawa H. Mechanisms governing autophagosome biogenesis. Nat. Rev. Mol. Cell Biol., 21, 439-458 (2020).

3) Kawano-Kawada M, Kakinuma Y, Sekito T. Transport of amino acids across the vacuolar membrane of yeast: its mechanism and physiological role. Biol. Pharm. Bull., 41, 1496-1501 (2018).

4) Kirkin V, Rogov VV. A diversity of selective autophagy receptors determines the specificity of the autophagy pathway. Mol. Cell, 76, 268-285 (2019)

5) Sánchez-Martín P, Komatsu M. p62/SQSTM1-steering the cell through health and disease. J. Cell Sci., 131, jcs222836 (2018).

6) Noda NN, Wang Z, Zhang H. Liquid-liquid phase separation in autophagy. J. Cell Biol., 219, e202004062 (2020).

7) Noda NN, Inagaki F. Mechanisms of autophagy. Annu. Rev. Biophys., 44, 101-122 (2015).

8) Yamaguchi M, Noda NN, Yamamoto H, Shima T, Kumeta H, Kobashigawa Y, Akada R, Ohsumi Y, Inagaki F. Structural insights into Atg10-mediated formation of the autophagy-essential Atg12Atg5 conjugate. Structure, 20, 1244-1254 (2012).

9) Sakoh-Nakatogawa M, Matoba K, Asai E, Kirisako H, Ishii J, Noda $\mathrm{NN}$, Inagaki F, Nakatogawa H, Ohsumi Y. Atg12-Atg5 conjugate enhances E2 activity of Atg3 by rearranging its catalytic site. Nat. Struct. Mol. Biol., 20, 433-439 (2013).

10) Zheng Y, Qiu Y, Grace CRR, Liu X, Klionsky DJ, Schulman BA. A switch element in the autophagy E2 Atg3 mediates allosteric regulation across the lipidation cascade. Nat. Commun., 10, 3600 (2019).

11) Hanada T, Noda NN, Satomi Y, Ichimura Y, Fujioka Y, Takao T, Inagaki F, Ohsumi Y. The Atg12-Atg5 conjugate has a novel E3like activity for protein lipidation in autophagy. J. Biol. Chem., 282, 37298-37302 (2007).

12) Noda NN, Ohsumi Y, Inagaki F. Atg8-family interacting motif crucial for selective autophagy. FEBS Lett., 584, 1379-1385 (2010).

13) Birgisdottir ÅB, Lamark T, Johansen T. The LIR motif-crucial for selective autophagy. J. Cell Sci., 126, 3237-3247 (2013).

14) Metlagel Z, Otomo C, Takaesu G, Otomo T. Structural basis of ATG3 recognition by the autophagic ubiquitin-like protein ATG12. Proc. Natl. Acad. Sci. U.S.A., 110, 18844-18849 (2013).

15) Yoshimoto K, Hanaoka $H$, Sato S, Kato $T$, Tabata S, Noda $T$, Ohsumi Y. Processing of ATG8s, ubiquitin-like proteins, and their deconjugation by ATG4s are essential for plant autophagy. Plant Cell, 16, 2967-2983 (2004).

16) Suzuki NN, Yoshimoto K, Fujioka Y, Ohsumi Y, Inagaki F. The crystal structure of plant ATG12 and its biological implication in autophagy. Autophagy, 1, 119-126 (2005).

17) Fujioka Y, Noda NN, Fujii K, Yoshimoto K, Ohsumi Y, Inagaki F. In vitro reconstitution of plant Atg8 and Atg12 conjugation systems essential for autophagy. J. Biol. Chem., 283, 1921-1928 (2008).

18) Otwinowski Z, Minor W. Processing of X-ray diffraction data collected in oscillation mode. Methods Enzymol., 276, 307-326 (1997).

19) Vagin A, Teplyakov A. Molecular replacement with MOLREP. Acta Crystallogr. D Biol. Crystallogr., 66, 22-25 (2010). 
20) Winn MD, Ballard CC, Cowtan KD, Dodson EJ, Emsley P, Evans PR, Keegan RM, Krissinel EB, Leslie AG, McCoy A, McNicholas SJ, Murshudov GN, Pannu NS, Potterton EA, Powell HR, Read RJ, Vagin A, Wilson KS. Overview of the CCP4 suite and current developments. Acta Crystallogr. D Biol. Crystallogr., 67, 235-242 (2011).

21) Emsley P, Lohkamp B, Scott WG, Cowtan K. Features and development of Coot. Acta Crystallogr. D Biol. Crystallogr., 66, 486-501 (2010).

22) Afonine PV, Grosse-Kunstleve RW, Echols N, Headd JJ, Moriarty NW, Mustyakimov M, Terwilliger TC, Urzhumtsev A, Zwart PH, Adams PD. Towards automated crystallographic structure refinement with phenix. refine. Acta Crystallogr. D Biol. Crystallogr., 68, 352-367 (2012)

23) Liebschner $\mathrm{D}$, Afonine $\mathrm{PV}$, Baker ML, et al. Macromolecular structure determination using X-rays, neutrons and electrons: recent developments in Phenix. Acta Crystallogr. D Struct. Biol., 75, 861-877 (2019).

24) Lovell SC, Davis IW, Arendall WB 3rd, de Bakker PI, Word JM, Prisant MG, Richardson JS, Richardson DC. Structure validation by Calpha geometry: phi, psi and Cbeta deviation. Proteins, 50, 437-450 (2003).

25) The PyMOL Molecular Graphics System, Version 2.0 Schrödinger, LLC.

26) Yamada Y, Suzuki NN, Hanada T, Ichimura Y, Kumeta H, Fujioka Y, Ohsumi Y, Inagaki F. The crystal structure of Atg3, an autophagy-related ubiquitin carrier protein (E2) enzyme that mediates Atg8 lipidation. J. Biol. Chem., 282, 8036-8043 (2007).

27) Taherbhoy AM, Tait SW, Kaiser SE, Williams AH, Deng A, Nourse A, Hammel M, Kurinov I, Rock CO, Green DR, Schulman BA. Atg8 transfer from Atg7 to Atg3: a distinctive E1-E2 architecture and mechanism in the autophagy pathway. Mol. Cell, 44, 451-461
(2011).

28) Qiu Y, Hofmann K, Coats JE, Schulman BA, Kaiser SE. Binding to E1 and E3 is mutually exclusive for the human autophagy E2 Atg3. Protein Sci., 22, 1691-1697 (2013).

29) Otomo C, Metlagel Z, Takaesu G, Otomo T. Structure of the human ATG12 ATG5 conjugate required for LC3 lipidation in autophagy. Nat. Struct. Mol. Biol., 20, 59-66 (2013).

30) Yamaguchi M, Matoba K, Sawada R, Fujioka Y, Nakatogawa H, Yamamoto H, Kobashigawa Y, Hoshida H, Akada R, Ohsumi Y, Noda NN, Inagaki F. Noncanonical recognition and UBL loading of distinct E2s by autophagy-essential Atg7. Nat. Struct. Mol. Biol., 19, 1250-1256 (2012).

31) Noda NN, Fujioka Y, Hanada T, Ohsumi Y, Inagaki F. Structure of the Atg12-Atg5 conjugate reveals a platform for stimulating Atg8PE conjugation. EMBO Rep., 14, 206-211 (2013).

32) Noda NN, Satoo K, Fujioka Y, Kumeta H, Ogura K, Nakatogawa $\mathrm{H}$, Ohsumi Y, Inagaki F. Structural basis of Atg8 activation by a homodimeric E1, Atg7. Mol. Cell, 44, 462-475 (2011).

33) Hong SB, Kim BW, Lee KE, Kim SW, Jeon H, Kim J, Song HK. Insights into noncanonical E1 enzyme activation from the structure of autophagic E1 Atg7 with Atg8. Nat. Struct. Mol. Biol., 18, $1323-1330$ (2011)

34) Sievers F, Wilm A, Dineen D, Gibson TJ, Karplus K, Li W, Lopez R, McWilliam H, Remmert M, Soding J, Thompson JD, Higgins DG. Fast, scalable generation of high-quality protein multiple sequence alignments using Clustal Omega. Mol. Syst. Biol., 7, 539 (2011).

35) Larkin MA, Blackshields G, Brown NP, Chenna R, McGettigan PA, McWilliam H, Valentin F, Wallace IM, Wilm A, Lopez R, Thompson JD, Gibson TJ, Higgins DG. Clustal W and Clustal X version 2.0. Bioinformatics, 23, 2947-2948 (2007). 